The ASTROPHYSICAL Journal, 285:L1-L3, 1984 October 1

(C) 1984. The American Astronomical Society. All rights reserved. Printed in U.S.A.

\title{
RELATIONS BETWEEN SPATIAL CORRELATIONS OF RICH CLUSTERS OF GALAXIES ${ }^{1}$
}

\author{
H. David Politzer and Mark B. Wise \\ California Institute of Technology \\ Received 1984 April 30; accepted 1984 June 22
}

\begin{abstract}
We express the $N$-point rich cluster correlation function in terms of the two-point rich cluster correlation function in a model where rich clusters formed wherever suitably averaged primordial energy density fluctuations are unusually large. The validity of our results is not restricted to regions where the connected (reduced) $N$-point correlation functions are small compared with unity.
\end{abstract}

Subject heading: galaxies: clustering

The two-point correlations of rich clusters of galaxies are observed to be much stronger than the two-point correlations of galaxies. For example, the two-point correlation function for Abell clusters $(R \geq 1)$ is unity at about $25 h^{-1} \mathrm{Mpc}$ while the two-point correlation for galaxies is unity at $5 h^{-1} \mathrm{Mpc}$. Furthermore the Abell cluster two-point correlation function increases with richness. The $R \geq 2$ clusters have stronger correlations than the $R=1$ clusters (Bahcall and Soneira 1983; Klypin, and Kopylov 1983; Hauser and Peebles 1973).

Recently Kaiser has suggested that the origin of this enhancement is essentially statistical (Kaiser 1984). If rich clusters formed where the primordial energy density fluctuations (averaged over an appropriate volume) are unusually large and the primordial fluctuations are Gaussian, then the rich cluster two-point correlation function is enhanced over the two-point energy density correlation function.

In this paper the $N$-point correlation functions for rich clusters are considered. We show that for Gaussian primordial fluctuations the $N$-point rich cluster correlation functions can be expressed in terms of the two-point rich cluster correlation function. Our results are valid even in regions where the connected (reduced) $\mathrm{N}$-point rich cluster correlation functions are about unity.

For Gaussian statistics the probability that the primordial energy density fluctuations, $\Delta \rho /\langle\rho\rangle$, take the value $\varepsilon(x)$ is

$$
P[\varepsilon(x)]=\frac{1}{Z} \exp \left[-\frac{1}{2} \int d x \varepsilon(x) K \varepsilon(x)\right],
$$

and the normalization factor $Z$ is given by the functional integral (Feynman and Hibbs 1965)

$$
Z=\int[d \varepsilon(\boldsymbol{x})] \exp \left[-\frac{1}{2} \int d \boldsymbol{x} \varepsilon(\boldsymbol{x}) K \varepsilon(\boldsymbol{x})\right] .
$$

Here $K$ is an operator that determines the spectrum of primordial energy density fluctuations. We are interested in

\footnotetext{
${ }^{1}$ Work supported in part by the U.S. Department of Energy under contract DE-AC03-81-ER40050.
}

density fluctuations averaged over the spatial volume relevant to rich cluster formation. Therefore, we introduce a filter function $W(\boldsymbol{y})$, which is essentially constant inside some radius and vanishes outside. This radius must be chosen to match what one identifies as a cluster. The two-point correlation of primordial energy density fluctuations averaged over such a volume is

$$
\begin{aligned}
\xi_{\rho}\left(\boldsymbol{x}_{1}-\boldsymbol{x}_{2}\right)= & \int[d \varepsilon(\boldsymbol{x})] \int d \boldsymbol{y}_{1} \varepsilon\left(\boldsymbol{x}_{1}-\boldsymbol{y}_{1}\right) W\left(y_{1}^{2}\right) \\
& \times \int d \boldsymbol{y}_{2} \varepsilon\left(\boldsymbol{x}_{2}-\boldsymbol{y}_{2}\right) W\left(y_{2}^{2}\right) P[\varepsilon(\boldsymbol{x})] .
\end{aligned}
$$

The integrations over $\boldsymbol{y}$ and $\boldsymbol{y}_{2}$ average the energy density fluctuations at $x_{1}$ and $x_{2}$ over the support of $W$. It is straightforward to show that

$$
\xi_{\rho}\left(\boldsymbol{x}_{1}-\boldsymbol{x}_{2}\right)=\int \frac{d \boldsymbol{k}}{(2 \pi)^{3}} \frac{e^{i \boldsymbol{k}\left(x_{1}-x_{2}\right)}}{\tilde{K}\left(k^{2}\right)} \tilde{W}\left(k^{2}\right)^{2} .
$$

Here $\tilde{W}$ is the Fourier transform of $W$, and $\tilde{K}$ is the operator $K$ in "momentum space" where it acts by multiplication. From equation (4) it is evident that $\tilde{K}$ is the inverse of the power spectrum. A Harrison-Zel'dovich spectrum (Harrison 1970: Zel'dovich 1972) has $\tilde{K}\left(k^{2}\right)$ proportional to $k^{-1}$ for wavelengths that crossed the horizon in the matter-dominated era.

The probability $P_{N}\left(x_{1}, \ldots, x_{N}\right)$ that at points $x_{1}, \ldots, x_{N}$ the energy density fluctuations averaged over a volume have a value greater than the threshold $t=\left[A \xi_{\rho}(0)\right]^{1 / 2}$ is

$$
\begin{aligned}
P_{N}\left(x_{1}, \ldots, x_{N}\right)= & \int_{t}^{\infty} d \alpha_{1} \cdots \int_{t}^{\infty} d \alpha_{N} \int[d \varepsilon(x)] P[\varepsilon(\boldsymbol{x})] \\
& \times \delta\left[\int d y \varepsilon\left(x_{1}-y\right) W\left(y^{2}\right)-\alpha_{1}\right] \cdots \\
& \times \delta\left[\int d y \varepsilon\left(x_{N}-y\right) W\left(y^{2}\right)-\alpha_{N}\right] .
\end{aligned}
$$


The integrals over $y$ remove short-wavelength fluctuations. The support of $W$ is chosen so that the fluctuations that reach the threshold $t$ have enough mass to be a rich cluster of galaxies. Using the representation $\delta(x)=1 / 2 \pi \int d \varphi e^{i \varphi x}$ for the delta functions in equation (5), the functional integral over $\varepsilon(\boldsymbol{x})$ is easily performed. We find that

$$
\begin{aligned}
P_{N}\left(x_{1}, \ldots, x_{N}\right)= & \frac{1}{(2 \pi)^{N / 2}(\operatorname{det} M)^{1 / 2}} \int_{t}^{\infty} d \alpha_{1} \cdots \int_{t}^{\infty} d \alpha_{N} \\
& \times \exp \left[-\frac{1}{2}\left(\alpha_{1}, \cdots \alpha_{N}\right) M^{-1}\left(\begin{array}{c}
\alpha_{1} \\
\vdots \\
\alpha_{N}
\end{array}\right)\right],
\end{aligned}
$$

where $M$ is the $N \times N$ matrix with entries

$$
[M]_{i j}=\xi_{\rho}\left(x_{i}-x_{j}\right) .
$$

Assuming that rich clusters formed wherever the energy density fluctuations $\varepsilon(x)$ averaged with $W$ over a suitable volume are greater than $\left[A \xi_{\rho}(0)\right]^{1 / 2}$ the $N$-point rich cluster correlation function is given by

$$
1+\xi_{\text {rc }}\left(x_{1}, \ldots, x_{N}\right)=\frac{1}{P_{1}^{N}} P_{N}\left(x_{1}, \ldots, x_{N}\right)
$$

The $N$-point rich cluster correlation function is easily evaluated when $A \gg 1$ and $\xi_{\rho}\left(x_{i}-x_{j}\right) / \xi_{\rho}(0) \ll 1$. Then we can use $\operatorname{det} M \approx\left[\xi_{\rho}(0)\right]^{N}$ and

$$
\left[M^{-1}\right]_{i j} \approx\left\{\begin{array}{cc}
\xi_{\rho}^{-1}(0) & i=j \\
-\xi_{\rho}\left(x_{i}-x_{j}\right) / \xi_{\rho}^{2}(0) & i \neq j
\end{array}\right.
$$

in equation (6). The integrals over $\alpha_{1}, \ldots, \alpha_{N}$ are performed using the leading term in their asymptotic expansion

$$
\int_{d}^{\infty} d x x^{p} e^{-(1 / 2) x^{2}} \approx d^{p-1} e^{-(1 / 2) d^{2}}\left[1+0\left(1 / d^{2}\right)\right] .
$$

These approximations result in the $\mathrm{N}$-point rich cluster correlation function

$$
\xi_{\mathrm{rc}}\left(\boldsymbol{x}_{1}, \cdots \boldsymbol{x}_{N}\right) \approx \exp \left[\sum_{i>j} \frac{A \xi_{\rho}\left(\boldsymbol{x}_{i}-\boldsymbol{x}_{j}\right)}{\xi_{\rho}(0)}\right]-1 .
$$

Note that equation (11) is valid as long as $A \gg 1$ and $\xi_{\rho}\left(x_{i}-x_{i}\right) / \xi_{\rho}(0) \ll 1$. However, the $N$-point correlation function for rich clusters of galaxies is not restricted to be small compared with unity.

Our expression for the two-point rich cluster correlation function

$$
\xi_{\mathrm{rc}}\left(\boldsymbol{x}_{1}, \boldsymbol{x}_{2}\right)=\exp \left[A \xi_{\rho}\left(\boldsymbol{x}_{1}-\boldsymbol{x}_{2}\right) / \xi_{\rho}(0)\right]-1
$$

agrees with that in (Kaiser 1984) when the rich cluster correlation function is small compared with unity.
If galaxies are good tracers of the mass distribution, then the galaxy two-point correlation $\xi_{g}$ satisfies

$$
\xi_{g}\left(x_{1}, x_{2}\right) \approx \xi_{\rho}\left(x_{1}-x_{2}\right) .
$$

The rich cluster correlations can then be expressed in terms of the galaxy two-point correlation function using equations (6)-(8). Of course, this only makes sense for cluster distances larger than the averaging radius of $W$, which Kaiser found to be approximately $9 h^{-1}$ Mpc from preliminary numerical simulations (Kaiser 1984). For these distances, the simple forms of equations (11) and (12) should be valid. In the circumstance that more than one large energy density fluctuation falls within a single averaging volume, this theoretical model will count them partially as a single cluster and partially as several. Hence, for distances not much greater than the chosen averaging radius, these formulae should be compared with similarly volume averaged observations.

The correlation of rich clusters with arbitrary energy density fluctuations can be expressed similarly. These are observable if the galaxy distribution traces the primordial energy density distribution. Then, for example, the galaxy rich cluster two-point correlation is

$$
\begin{aligned}
\xi_{g-\mathrm{rc}}\left(\boldsymbol{x}_{1}, \boldsymbol{x}_{2}\right)= & \frac{1}{P_{1}} \int_{t}^{\infty} d \alpha \int[d \varepsilon(\boldsymbol{x})] \int d \boldsymbol{y} \varepsilon\left(x_{1}-\boldsymbol{y}\right) W\left(y^{2}\right) \\
& \times \delta\left[\int d \boldsymbol{y} \varepsilon\left(\boldsymbol{x}_{2}-\boldsymbol{y}\right) W\left(y^{2}\right)-\alpha\right] P[\varepsilon(\boldsymbol{x})] \\
\approx & {\left[\frac{A}{\xi_{g}(0)}\right]^{1 / 2} \xi_{g}\left(\boldsymbol{x}_{1}, \boldsymbol{x}_{2}\right), }
\end{aligned}
$$

where the simple approximate form holds when $A \gg 1$ and $\xi_{g}\left(x_{1}, x_{2}\right) \ll \xi_{g}(0)$. The observed galaxy rich cluster correlation is indeed roughly the geometric mean of the galaxygalaxy and the cluster-cluster correlations (Hauser and Peebles 1973).

Using equation (12) we can express the $N$-point correlation function for rich clusters in terms of the two-point correlation function for rich clusters

$$
\xi_{\mathrm{rc}}\left(x_{1}, \ldots, x_{N}\right)=\left\{\prod_{i>j}\left[\xi_{\mathrm{rc}}\left(\boldsymbol{x}_{i}, \boldsymbol{x}_{j}\right)+1\right]\right\}-1 .
$$

Equation (15) is our main result. It does not depend on galaxies being good tracers of the mass distribution. Furthermore, equation (15) is valid even in regions where the rich cluster two-point correlation function is not small compared with unity. So the nontrivial connected (reduced) higher correlations predicted by equation (15) may be observable. For example, the connected (reduced) three-point rich cluster correlation function is

$$
\begin{aligned}
\xi_{\mathrm{rc}}^{c}\left(x_{1}, x_{2}, x_{3}\right)= & \xi_{\mathrm{rc}}\left(x_{2}, x_{1}\right) \xi_{\mathrm{rc}}\left(x_{3}, x_{1}\right) \\
& +\xi_{\mathrm{rc}}\left(x_{2}, x_{1}\right) \xi_{\mathrm{rc}}\left(x_{3}, x_{2}\right) \\
& +\xi_{\mathrm{rc}}\left(x_{3}, x_{1}\right) \xi_{\mathrm{rc}}\left(x_{3}, x_{2}\right) \\
& +\xi_{\mathrm{rc}}\left(x_{3}, x_{2}\right) \xi_{\mathrm{rc}}\left(x_{3}, x_{1}\right) \xi_{\mathrm{rc}}\left(x_{2}, x_{1}\right)
\end{aligned}
$$


Observation of the connected three-point rich cluster correlation function given in equation (16) would support the hypothesis that rich clusters arise wherever Gaussian primordial energy density fluctuations, averaged over a suitable volume, are unusually large. As noted in Kaiser (1984), this hypothesis has important ramifications for the spectrum of primordial energy density fluctuations that give rise to the large-scale structure of the universe.

In the new inflationary cosmology (Guth 1981; Linde 1982; Albrecht and Steinhardt 1982) the fluctuations have Gaussian statistics (Abbott and Wise 1984). However, the scale invariant spectrum of fluctuations predicted by the new inflationary cosmology (Guth, and Pi 1982; Bardeen, Steinhardt, and Turner 1983; Starobinskii 1982; Hawking 1982) is not compatible with positive two-point correlations at very large distances. If the statistics of the underlying energy density fluctuations on the relevant distance scales deviate slightly from Gaussian, then our formulae are approximate, with small corrections. For example, the primordial energy density may have a connected (reduced) three-point correlation, $\xi_{\rho}^{c}$, characterized by a small' parameter $\lambda$, e.g.

$$
\xi_{\rho}^{c}\left(x_{1}, x_{2}, x_{3}\right)=\lambda \int d x \xi_{\rho}\left(x-x_{1}\right) \xi_{\rho}\left(x-x_{2}\right) \xi_{\rho}\left(x-x_{3}\right) .
$$

Then the rich cluster two-point and three-point correlation functions get correction factors of order $\left[A / \xi_{\rho}(0)\right]^{3 / 2} \xi_{\rho}^{c}$. At separations, $d$, for which the $\lambda=0$ rich cluster correlation functions are order unity, these corrections are systematically smaller than $\lambda d^{3}$ by $A^{-3 / 2}$.

If the universe has a critical density, the galaxies themselves may have formed only at hot spots of an essentially Gaussian primordial energy density distribution. Then all galaxy correlations would be predicted in terms of the galaxy two-point function, as given by equation (15). This attractive picture has two major problems. While most of the matter of the universe would be spread thin in intergalactic space, there is a distinct absence of observed hydrogen absorption lines associated with these regions (Gunn and Peterson 1965). Second, the observed galaxy three-point correlation (Peebles 1980) does not show a cubic term as is predicted by equation (16). However, these obstacles may be surmountable. Perhaps intergalactic hydrogen is all ionized. Also, on the scale at which galaxy correlations are large, there has likely been sufficient time for gravitational nonlinearities to act to rearrange the galaxy distribution. So the basic picture may be correct while its simplest consequences are only immediately apparent on somewhat longer distance scales.

We thank C. Hogan for many useful discussions.

\section{REFERENCES}

Abbott, L., and Wise, M. 1984, Phys. Letters, 135B, 279.

Albrecht, A., and Steinhardt, P. 1982, Phys. Rev. Letters, 48, 1220

Bahcall, N., and Soneira, R. 1983, Ap. J., 270, 20.

Bardeen J., Steinhardt, P., and Turner, M. 1983, Phys. Rev., D28, 679.

Feynman, R., and Hibbs, A. 1965, Quantum Mechanics and Path Integrals

(New York: McGraw Hill).

Gunn, J., and Peterson, B. 1965, Ap. J., 142, 1633.

Guth, A. 1981, Phys. Rev., D23, 347.

Guth, A., and Pi, S. Y. 1982, Phys. Rev. Letters, 49, 1110

Harrison, E. 1970, Phys. Rev., D1, 2726.
Hauser, M., and Peebles, P. 1973, Ap. J., 185, 757.

Hawking, S. 1982, Phys. Letters, 115B, 295.

Kaiser, N. 1984, Ap. J (Letters), 284, L9.

Klypin, A., and Kopylov, A. 1983, Soviet Astr. Letters, 9, 41.

Linde, A. 1982, Phys. Letters, 108B, 389

Peebles, P. J. E. 1980, The Large Scale Structure of the Universe (Princeton: Princeton University Press)

Starobinskii, A. 1982, Phys. Letters, 117B, 175.

Zel'dovich, Y. 1972, M.N.R.A.S., 160,1P.

H. David Politzer and Mark B. WiSE: Charles C. Lauritsen Laboratory of High Energy Physics, 452-48, Pasadena, CA 91125 